\title{
Creating Smart and Adaptable Medical Devices: Embedding Miniaturized Biosensors
}

\section{Bruce Reiner $I^{*}$}

Department of Radiology, Veterans Affairs Maryland Healthcare System, 10 North Greene Street, Baltimore, Maryland, USA

*Corresponding author: Bruce Reiner I, Department of Radiology, Veterans Affairs Maryland Healthcare System, 10 North Greene Street, Baltimore, Maryland, USA, Tel: 443-513-4201; E-mail: breiner1@comcast.net

Received date: July 11, 2017; Accepted date: July 23, 2017; Published date: July 30, 2017

Copyright: () 2017 Bruce Reiner I. This is an open-access article distributed under the terms of the Creative Commons Attribution License, which permits unrestricted use, distribution and reproduction in any medium, provided the original author and source are credited.

\section{Creating Adaptive Medical Devices}

A "smart" device is defined as an electronic device generally connected to other devices or networks via different protocols (e.g. BlueTooth, WiFi) that can operate to some extent interactively and autonomously [1]. In this sense, the word "smart" refers to computing interoperability and not the inherent functionality of the device or derived data. When referring to medical device performance and analysis, a preferred term would be "intelligent" device, which is defined as any type of equipment that has its own computing capability [1]. Based upon these definitions, an alternative terminology for describing new trends in medical device technology may be that of "adaptive"; for it connotes the ability of the medical device to proactively adjust to new conditions or measures.

A wide array of "smart" medical applications have been created to date, which transcend multiple organ systems and pathologies. These can range from relatively simplistic applications monitoring physiologic measurements (e.g. temperature, heart rate, oxygen saturation, respiratory rate) to complex applications serving both diagnostic and therapeutic functions (e.g. artificial pancreas). While the majority of these devices are stationary in positioning, a few function through locational change (e.g. capsule endoscopy); thereby providing dynamic data in a variety of anatomic locations. While these "smart" devices offer significant advancements in diagnosis and treatment; they do remain limited responsiveness to the local environment in which they reside. For implanted devices which are designed to be longer term in duration, this can become problematic since the devices are foreign bodies, and as such are prone to a variety of complications (e.g. infection, thrombosis).

In the course of innovation for the next generation of smart medical devices, one possibility is to redefine the responsiveness and adaptability of devices, from a passive to more proactive role. In doing so, the device takes on a more dynamic role within its local environment, while maintaining its inherent functionality. In addition to monitoring and responding to local environmental change, another potential adaptive feature is the ability to self- diagnose device structural integrity. As an example, an "adaptive" vascular stent could serve its primary function of maintaining arterial patency while also continuously monitoring structural integrity of the stent (e.g. leakage), flow characteristics (e.g. directionality, velocity), cellular physiology (e.g. platelet aggregation in the formation of thrombus), and chemistry (e.g. cytokines related to infection). The additional knowledge gained by this continuous collection and analysis of in vivo data not only creates newfound knowledge of device performance, but also provides a valuable means for early intervention, in the event of device malfunction or concomitant pathology.
A number of recent technologic and informational advancements provide the capability for this proposed innovation. From the technologic perspective increased computer power, hardware miniaturization, advance software capability, and network connectivity offer new technical opportunities. From the informational standpoint large data storage capacity, creation of referenceable databases, artificial intelligence, and computerized data analytics provide enhanced diagnostic capabilities. The centre piece of such an innovation is built around miniaturized biosensors, which provide a unique opportunity to expand device functionality, data analytics, and early diagnosis and treatment.

\section{Embedded Miniaturized Biosensors}

Recent advances in biomedical engineering has led to the creation of miniaturized biosensors (i.e., micro or miniature total analysis systems), which are commonly referred to as "lab on a chip "devices. [2]. These miniaturized biosensors provide a number of advantages when applied to in vivo medical monitoring including reduced size, small sample volumes, multi-analyte detection, reduced analysis time, reduced reagent use, and high uniformity [3]. These advanced tools for studying cellular physiology and pathology provide the medical community with advanced disease diagnosis and treatment, not achievable by traditional methods.

The current state of the art in miniaturized medical biosensors involve micro-electro-mechanical systems (MEMS), which are mechanical and electromechanical elements developed through micro fabrication techniques. MEMS technologies have rapidly progressed over time to currently establish a wide range of small high performance and inexpensive sensors able to detect and respond to a wide array of physical variables including (but not limited to) pressure, position, motion, strain, radiation, and flow [4].

MEMS sensors can be integrated with information and communication technologies to create wireless communication and sensor networks; enabling the creation of compact, high performance, low power, low cost solutions for a wide range of applications [5-9]. Another key feature of MEMS technology is the ability to merge quantitative measurements with embedded intelligence.

In recent years, Biomedical or Biological Micro-Electro-Mechanical Systems (BioMEMS) have shown a number of promising potential clinical applications related to advanced diagnosis, therapy, and tissue engineering. In the area of bio-molecular analysis, BioMEMS provide an opportunity for sensing microorganisms, DNA strands, molecules, viruses, and cells [10]. Motion sensors (e.g. accelerometers and gyroscopes) can be used in the analysis of motor impairment disorders (e.g. Parkinson's disease), to provide objective analysis of motion abnormalities, which can in turn be used for enhanced diagnosis and 
treatment assessment. BioMEMS have also been used for advance tissue engineering applications. Examples include complementary metal-oxide semiconductor (CMOS) compatible MEMS technology targeting label-free selective detection of biomolecules (i.e., specific RNA sequences) [11] and BioMEMS device (based on a silicon dioxide-silicon nitride structure) used for testing mechanical properties of living cells [12]. MEMS devices also possess drug delivery capabilities through the incorporation of microreservoir drug depots, micropumps, valves, and sensors [13].

To date, the primary focus of BioMEMS in medicine have focused on the monitoring of chronic disease (e.g. hypertension, obesity, COPD, diabetes, heart failure). While still in its infancy, the estimated market for MEMS sensors in medical disease monitoring and diagnosis is forecast to reach 6 billion dollars by 2018 [14]. The proposed application of incorporating BioMEMS into medical device technology attempts to expand the purview of BioMEMS while providing new and currently lacking adaptive capabilities to implanted medical devices.

\section{Data Requirements}

Data requirements for an "adaptable" medical device should be context specific; and consist of a combination of device-specific functional, structural, and pathologic data. In the example of a vascular catheter, device specific functional data would be related to vascular flow (e.g. pressure, velocity, turbulence, directionality). Structural data would include data related to the structural integrity of the device and its individual components (e.g. wall permeability, surface defects, breakage, aperture patency). Pathologic data would be related to commonly encountered disease processes specific to the device and its local anatomy (e.g. bleeding, cellular proliferation, infection, thrombus).

The derived data would be created in a standardized format to provide for the creation of referenceable databases; which can in turn be used to create context specific analytics.

One highly publicized example is the artificial pancreas, which provides continuous glucose level monitoring and insulin release (references); providing both diagnostic and therapeutic purposes.

A number of demographic, economic, technical, and clinical factors are driving the creation of "smart" medical devices including the aging patient population, skyrocketing healthcare costs, shift from therapeutic to preventative medicine, technologic advancements in micro and nanotechnology.

A number of technologic and informational advances have the capabilities of dramatically transforming medical device innovation. From the technologic perspective increased computer power, hardware miniaturization, advance software capability, and network connectivity offer new technical opportunities. From the informational standpoint large data storage capacity, creation of large referenceable databases, artificial intelligence, and computerized data analytics provide enhanced diagnostic capabilities. Collectively, these provide synergistic opportunities to facilitate the practices of personalized and evidence based medicine.

The creation of "adaptable" medical devices would in theory create a mechanism by which the devices themselves become proactively engaged in real-time diagnosis and treatment. While most "smart" devices to date are external or superficially located within the body, the concept can be extended to a wide array of internally located "in vivo" devices, which up to now have largely been functional in nature and devoid of active data collection and analysis. One of the most pressing challenges (and innovation opportunities) for contemporary medical devices are the myriad of complications they routinely experience (e.g. infection, thrombus, mechanical breakdown). If one could effectively create intelligent and adaptable medical devices with the inherent capabilities of recording, tracking, and analyzing data specific to these complications; one could in theory improve the lifetime and functionality of the device, while simultaneously reducing patient morbidity and mortality.

\section{The Biofilm Conundrum}

Medical devices and biofilms go hand and hand, it's not a question of if but instead when. Essentially all medical devices at some point in time will be subjected to biofilm formation; which is a process where microorganisms irreversibly attach to and grow on a surface. Once adherence takes place, extracellular polymers are produced which facilitate attachment and matrix formation, resulting in an alteration in the phenotype of the microorganisms with respect to growth rate and gene transcription [15].

Biofilms have great significance to public health, because biofilmassociated micro-organisms exhibit dramatically decreased susceptibility to antimicrobial agents [16]. This decreased susceptibility may be intrinsic (i.e., as a natural outcome of growth in the biofilm) or acquired (due to transfer of extra chromosomal elements to susceptible organisms in the biofilm). The net result is that conventional antibiotics therapy is rarely effective long term, and frequently results in systemic infection and medical device removal.

The clinical ramifications of medical device biofilms has been well documented and encompasses a wide array of medical devices and organ systems. The US National Institutes of Health estimates that over $80 \%$ of all microbial infections in humans are caused by biofilms [5]. While medical device associated infections are ubiquitous, most available data has focused on the critical ill patient population which by the very nature of their clinical status routinely have medical devices. In this patient population, the most common site of infection is the urinary tract, with $95 \%$ of infections determined to be catheter related and affecting approximately 450,000 patients annually in U.S. hospitals $[17,18]$. An equally concerning source of medical device infections are vascular catheters, which account for $87 \%$ of bloodstream infections [8]. In the US alone, over 5 million central venous catheters are inserted annually, resulting in over 200,000 bloodstream infections per year [19]. Each individual central venous catheter associated bloodstream infection has been estimated to add over $\$ 45,000$ of cost to healthcare delivery; with the cumulative cost of the 5 major types of medical device related infections estimated to be $\$ 9.8$ billion annually in the U.S. [20].

While these infection rates are staggering, they may in actuality be underestimated due to the relative failure of conventional microbiological techniques to accurately culture organisms in the biofilm [21].

\section{Pathophysiology: Understanding the Challenge Ahead}

Biofilm development can be divided into three stages; attachment of cells to a surface (i.e., adherence), growth of the cells into a sessile biofilm colony (i.e., maturation), and detachment of cells from the colony into the surrounding medium (i.e., dispersal). The first step of biofilm formation involves cellular adherence to the underlying 
medical device surface; which involves both reversible and irreversible stages. The characteristics of the underlying surface of attachment may have a significant impact on the rate and extent of cellular attachment. In general, rougher and more hydrophobic materials tend to develop biofilms more rapidly $[22,23]$. When medical devices are placed in a fluid environment (e.g. blood, urine, cerebrospinal fluid), a conditioning film or coating takes place on the device surface which may confer chemical properties to the surface modifying cellular adherence. Other local dynamic forces may also play a role in adherence including hydrodynamic forces, repulsive forces, and nutrient availability [24].

Once cells irreversibly attach to a medical device surface they will begin cell division forming micro- colonies and produce extracellular polymers. These extracellular polymeric substances consist primarily of polysaccharides and provide the matrix or essential structure of the biofilm. They are tenaciously bound to the underlying surface, are heterogeneous in nature, and develop internal water channels over time which provide for transport of nutrients and oxygen to cells within the biofilm [14]. Over time, the biofilm entraps a variety of particles and cells including fibrin, red blood cells, and platelets.

Biofilm growth confers a marked decrease in sensitivity to antimicrobials (e.g. antibiotics), which may be due to the intrinsic protective effects of the biofilm matrix or acquired protection through the transfer of antibiotic resistant plasmids. Plasmids are extra chromosomal circles of DNA which may encode resistance to antibiotics. The rates of plasmid transfer between microorganisms has been shown to be several orders of magnitude higher in biofilms [25]. In addition, biofilm associated microorganisms tend to have reduced growth rates, thereby minimizing the biologic potential for antimicrobial agents to affect inactivation kinetics [26].

The final stage in the biofilm life cycle is dispersal, which enables biofilms to spread and colonize new surfaces. Dispersal can occur through either passive or active means. Passive dispersal represents cell detachment caused by external forces including fluid shear, abrasion, and human intervention [27]. Active dispersal refers to mechanisms mediated by the cells, often in response to the local environment. Examples of active dispersal include enzymes (e.g. dispersion B, deoxyribonuclease) which degrade the biofilm extracellular matrix, nutrient availability, fluctuations in oxygen levels, presence of toxins, and other stress inducing conditions [28-30].

\section{Intervention Strategies and Innovation Opportunity}

Conventional intervention strategies primarily focus on medical device surface components and antibiotic therapy. Medical device microbial adhesion depends on the physicochemical properties of the device surface components. Given the hydrophobic nature of microbial surfaces, one strategy for prevention of bacterial adhesion is to coat the medical device with hydrophilic polymers. A variety of hydrophilic coatings have been used including hydrophilic polymers (e.g. hyaluronic acid), silver based polymers, hydrogel coatings, heparin coatings or bindings [31-34].

Antibiotic coating of medical devices is a commonly deployed technique but has been found to be of limited value, due to a number of factors including poor penetration of the biofilm matrix, transfer of antibiotic resistance via plasmids, high incidence of poly microbial infections, and decreased metabolic state of biofilm bacteria decreasing antibiotic susceptibility $[35,36]$. Initial design of antibiotic impregnated medical devices (e.g. vascular catheters) featured a burst release of the antibiotic in the first few hours followed by a long lasting slow release at low concentrations, which proved ineffective. An alternative strategy has been more recently developed utilizing longer lasting antibiotic release which is dependent on the catheter polymers to absorb larger amounts of antibiotics and release them over a longer period of time at relatively constant concentrations [37]. At the same time, the poly microbial nature of biofilm infections require combination drug use with different antimicrobial spectra and modes of action.

While a variety of other intervention strategies have been attempted, the overall degree of success of these therapies has been lacking, as evidenced by the high infection rates of medical devices; which frequently results in device removal, sepsis, and on occasion death. An alternative approach is to transform the medical device from a passive to proactive role; in which the device incorporates biosensor technology to actively record data for the purpose of continuously monitoring and quantifying the presence of microorganisms and other cellular aggregates on its surface. In addition to real-time diagnosis, these embedded biosensors can also serve therapeutic purposes through a variety of mechanical and chemical processes; aimed at prohibiting adherence and aggregation. By incorporating biosensor technology directly within the device walls, the derived data can be used to create reference able databases; which can in turn be correlated with other data contained within the patient healthcare record. The real-time prospective data can be used to create patient and device specific "profiles", which can serve as an early warning sign or computerized alert in the event that data falls outside the baseline range of "normalcy". When this longitudinal diagnostic data is correlated with intervention and outcomes data; customizable best practice guidelines can be created relating to device selection, individual patient attributes, clinical context, and treatment options.

The device embedded biosensors can be incorporated into a wireless network, which provides the ability to transmit biosensor derived data to local and remote data storage units. At the same time, multiple devices within a single individual patient can be linked via this wireless network to provide inter-device data communication and analysis. The net effect is to create a series of "smart", "intelligent" and "adaptable" devices within a single patient; which provides for longitudinal devicespecific data collection, analysis, and intervention. The specifics related to device construction, biosensor integration, database creation and analysis, and network communication will be discussed in subsequent articles.

\section{Conclusion}

In the Art of War, the famous Chinese military strategist Sun Tzu stated "The supreme art of war is to subdue the enemy without fighting". Applying this analogy to biofilms, one would postulate that the most effective method of combatting medical device biofilm formation (and subsequent infection) is to prevent the biofilm from forming in the first place. This would entail prevention of microbial surface adhesion and colonization. If this can be effectively accomplished, then the more difficult steps of interference with signal molecules modulating biofilm formation and disaggregation of the biofilm matrix can be avoided.

Medical device microbial adhesion depends on the physicochemical properties of the device surface components. Given the hydrophobic nature of microbial surfaces, one strategy for prevention of bacterial adhesion is to coat the medical device with hydrophilic polymers. A variety of hydrophilic coatings have been used including hydrophilic 
polymers (e.g. hyaluronic acid), silver based polymers, hydrogel coatings, heparin coatings or bindings [38,39].

Antibiotic coating of medical devices is a commonly deployed technique but has been found to be of limited value, due to a number of factors including poor penetration of the biofilm matrix, transfer of antibiotic resistance via plasmids, high incidence of poly microbial infections, and decreased metabolic state of biofilm bacteria decreasing antibiotic susceptibility [40]. Initial design of antibiotic impregnated medical devices (e.g. vascular catheters) featured a burst release of the antibiotic in the first few hours followed by a long lasting slow release at low concentrations, which proved ineffective. An alternative strategy has been more recently developed utilizing longer lasting antibiotic release which is dependent on the catheter polymers to absorb larger amounts of antibiotics and release them over a longer period of time at relatively constant concentrations [19]. At the same time, the poly microbial nature of biofilm infections require combination drug use with different antimicrobial spectra and modes of action.

While antibiotics to date represent the most commonly deployed strategy for treating biofilm infections, a number of alternative synergistic strategies have been attempted with varying degrees of success. In vivo experiments have shown the application of an electric current can enhance the activity of certain antibiotics (i.e., bioelectric effect) [41]. Other examples of antimicrobial synergy have been reported with ultrasound, light, and nanoparticles. Ultrasound waves combined with gentamycin entrapped in bone cement was reported to reduce by $70 \%$ biofilm formation in a rabbit model [27]. DiPoto et al. [28] reported a significant reduction in Staphylococcus aureus cells in biofilm when simultaneously exposed to a photosensitization drug and visible light. This strategy of photodynamic treatment is based on the combined action of visible light and a photosensitizer drug that generates cytotoxic reactive oxygen species and free radicals that are bactericidal. Another novel strategy employs the use of nanoparticles which can be guided to the region of antibiotic release through the application of a magnetic field.

\section{References}

1. Daved W (2013) Definition of smart device.

2. Ul Haque A, Rokkam M, Decarlo AR, Wereley ST, Roux SJ, et al. (2007) A MEMS fabricated call electrophysiology biochip for in silico calcium measurements. Sensors and Actuators 123: 391-399.

3. Suzuki H (2000) Advances in microfabrication of electrochemical sensors and systems. Electroanalysis 12: 703-715.

4. Ciuti G, Ricotti L, Menciassi A, Dario P (2015) MEMS sensor technologies for human centered applications in healthcare, physical activities, safety, and environmental sensing: A review on research activities in Italy. Sensors 15: 6441-6468.

5. Magno M, Benini L, Spagnol C, Popovici E (2013) Wearable low power dry surface wireless sensor nodes for healthcare monitoring applications. In Proceedings of the 2013 IEEE 9th international Conference on Wireless Mobile Computing, Networking and Communications (WiMOB), Lyon France. pp: 189-195.

6. Ferrari M, Lee AP, Lee J (2007) BioMEMS and biomedical nanotechnology. Volume 1: Biological and Biomedical Nanotechnology.

7. Tedeschi L, Domenici C, Russino V, Nannini A, Pieri F (2014) Label-free detection of specific RNA sequences by a DNA-based CMOS BioMEMS. Sensors and Microsystems. Springer: Berlin, Germany. pp: 277-280.

8. Fior R, Maggiolino S, Lazzarino M, Sbaizero O (2011) A new transparent BioMEMS for uniaxial single cell stretching. Microsyst Technol 17: 1581-1587.
9. Chan M, Esteve D, Fourniols JY, Escriba C, Campo E (2012) Smart wearable systems: Current status and future challenges. Artificial Intelligence in Medicine 56: 137-156.

10. http://internetofthingsagenda.techtarget.com/defintion/intelligent-device

11. Donlan RM (2001) Biofilm formation: A clinically relevant microbiological process. Clin Infect Dis 33: 1387-1392.

12. Stewart PS, Costerton JW (2001) Antibiotic resistance of bacteria in biofilms. Lancet 358: 135-138.

13. Davies D (2003) Understanding biofilm resistance to antimicrobial agents. Nature Reviews Drug Discovery 2: 114-122.

14. Klevens RM, Edwards JR, Richards CL, Horan TC, Gaynes RP, et al. (2007) Estimating healthcare associated infections and deaths in US hospitals. Public Health Rep 122: 160-166.

15. Richards MJ, Edwards JR, Culver DH, Gaynes RP (1999) Nosocomial infections in medical intensive care units in the United States. National Nosocomial Infections Surveillance System. Crit Care Med 27: 887-892.

16. Mermel LA, Farr BM, Sheretz RJ Raad II, O'Grady N, et al. (2001) Guidelines for the management of intravascular catheter-related infections. Clin Infect Dis 32: 1249-1272.

17. Zimlichman E, Henderson D, Tamir O, Franz C, Song P, et al. (2013) Healthcare associated infecitons: A meta-analysis of costs and financial impact on the US healthcare system. JAMA Int Med 173: 2039-2046.

18. Weinstein RA, Darouiche RO (2001) Device-associated infections: A macroproblem that starts with microadherence. Clin Infect Dis 33: 1567-1572.

19. Strachan CJL (1993) Antibiotic prophylaxis in peripheral vascular and orthopedic prosthetic surgery. J Antimicrob Chemother 31: 65-78.

20. Fletcher M, Loeb G (1979) Influence of substratum characteristics in the attachment of a marine pseudomonad to solid surfaces. Appl Environ Microbiol 37: 67-72.

21. Pringle JH, Fletcher M (1983) Influence of substratum wettability on attachment of freshwater bacteria to solid surfaces. Appl Environ Microbiol 45: 811-817.

22. Kostakioti M, Hadjifrangiskou M, Hultgren SC (2013) Bacterial biofilms: Development, dispersal, and therapeutic strategies in the dawn of the postantibiotic era. Cold Spring Harbor Perspect Med 3: 1-24.

23. Lewandowski Z (2000) Structure and function of biofilms. In: Evans LV, editor. Biofilms: recent advances in their structure and control. Harwood Academic Publishers, Amsterdam. pp: 1-17.

24. Christensen BB, Sternberg C, Andersen JB, Andersen JB, Eberl L, et al. (1998) Establsihment of new genetic traits in a microbial biofilm community. Appl Environ Microbiol 64: 2247-2255.

25. Kaplan JB (2010) Biofilm dispersal. J Dent Res 3: 205-218.

26. Karatan E, Watnick P (2009) Signals, regulatory networks, and materials that build and break bacterial biofilms. Microbiol Mol Biol Rev 73: 310-347.

27. Hong SH, Lee J, Wood TK (2010) Engineering global regulator Hha of E.coli to control biofilm dispersal. Microb Biotechnol 3: 717-728.

28. Francolini I, Donelli G (2010) Prevention and control of biofilm-based medical device infections. Pathogens and Disease 59: 227-238.

29. Monteiro DR, Gorup LF, Takamiya AS, Ruvollo-Filho AC, de Camargo ER, et al. (2009) The growing importance of materials that prevent microbial adhesion: Antimicrobial effect of medical devices containing silver. Journal of Antimicrobial Agents 34: 103-110.

30. Bjarnsholt T, Givskov M (2008) Quorum sensing inhibitory drugs as next generation antimicrobials: Worth the effort? Curr Infect Dis Rep 10: 22-28.

31. Kaufmann GF, Park J, Janda KD (2008) Bacterial quorum sensing: A new target for anti-infective immunotherapy. Expert Opin Biol Th 8: 719-724.

32. Martin CA, Hoven AD, Cook AM (2008) Therapeutic frontiers: Preventing and treating infectious disease by inhibiting bacterial quorum sensing. Eur J Clin Microbiol 27: 635-642.

33. Costerdon JW, Stewart PS, Greenberg EP (1999) Bacterial biofilms: A common cause of persistent infections. Science 284: 1318-1322. 
Citation: Bruce RI (2017) Creating Smart and Adaptable Medical Devices: Embedding Miniaturized Biosensors. J Bioengineer \& Biomedical Sci 7: 232. doi:10.4172/2155-9538.1000232

Page 5 of 5

34. Ehrlich GD, Stoodley P, Kathu S, Zhao Y, McLeod BR, et al. (2005) Engineering approaches for the detection and control of orthopedic biofilm infections. Clin Orthop Relat R 437: 59-66.

35. Del Pozo JL, Rouse MS, Mandrekar JN, Steckelberg JM, Patel R (2009) The electrocidal effect: Reduction of Staphylococcus and Pseudomonas biofilms by prolonged exposure to low intensity electrical current Antimicrob Agents Ch 53: 41-45.

36. Ensing GT, Roeder BL, Nelson JL, Van Horn JR, Van der Mei HC, et al. (2005) Effect of pulsed ultrasound in combination with gentamycin on bacterial viability in biofilms on bone cement in vivo. J Appl Microbiol 99: 443-448.

37. Di Poto A, Sbarra MS, Provenza G, Visai L, Speziale P, et al. (2009) The effect of photodynamic treatment combined with antibiotic action on host defense mechanisms on Staphylococcus aureus biofilms. Biomaterials 30: 3158-3166.

38. Corchero JL, Villaverde A (2009) Biomedical applications of distally controlled magnetic nanoparticles. Trends Biotechnol 27: 468-476.

39. Xie J, Huang J, Li X, Sun S, Chen X, et al. (2009) Iron oxide nanoparticle platform for biomedical applications. Curr Med Chem 16: 1278-1294.

40. Donelli G, Francolini I, Romoli D, Guaglianone E, Piozzi A, et al. (2007) Synergistic activity of dispersin B and cefamandole nafate in inhibition of Staphylococcl biofilm growth on polyurethanes. Antimicrob Agents Ch 51: 2722-2740.

41. Chaignon P, Sadouskaya I, Ragunah C, Ramasubbu N, Kaplan JB, et al. (2007) Susceptibiltiy of Staphylococcal biofilms to enzymatic treatments depends on their clinical composition. App Microbiol Biot 75: 125-132. 